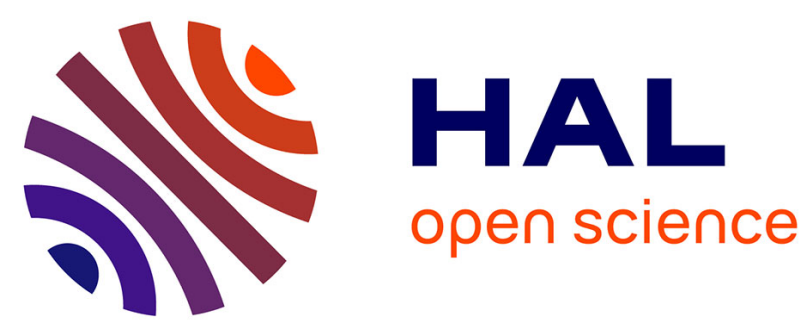

\title{
How do walkers behave when crossing the way of a mobile robot that replicates human interaction rules?
}

Christian Vassallo, Anne-Hélène Olivier, Philippe Souères, Armel Crétual, Olivier Stasse, Julien Pettré

\section{- To cite this version:}

Christian Vassallo, Anne-Hélène Olivier, Philippe Souères, Armel Crétual, Olivier Stasse, et al.. How do walkers behave when crossing the way of a mobile robot that replicates human interaction rules? Gait \& Posture, 2018, 60, pp.188-193. 10.1016/j.gaitpost.2017.12.002 . hal-01717722

HAL Id: hal-01717722

https://hal-univ-rennes1.archives-ouvertes.fr/hal-01717722

Submitted on 29 Mar 2018

HAL is a multi-disciplinary open access archive for the deposit and dissemination of scientific research documents, whether they are published or not. The documents may come from teaching and research institutions in France or abroad, or from public or private research centers.
L'archive ouverte pluridisciplinaire HAL, est destinée au dépôt et à la diffusion de documents scientifiques de niveau recherche, publiés ou non, émanant des établissements d'enseignement et de recherche français ou étrangers, des laboratoires publics ou privés. 


\section{How do walkers behave when crossing the way of a mobile robot that replicates human interaction rules?}

Christian Vassallo ${ }^{a}$, Anne-Hélène Olivier ${ }^{b, c}$, Philippe Souères ${ }^{a}$, Armel Crétual ${ }^{b c}$, Olivier Stasse ${ }^{a}$ and Julien Pettréc

${ }^{a}$ CNRS, LAAS, 7 Avenue du colonel Roche, F-31400 Toulouse, France

${ }^{\mathrm{b}}$ Univ de Toulouse, LAAS, F-31400 Toulouse, France

c M2S lab, University Rennes 2 - ENS Rennes - UEB, Avenue Robert Schuman, Campus de Ker Lann, 35170 Bruz, France

d Inria Rennes, Centre de Rennes Bretagne Atlantique, Campus universitaire de Beaulieu, 35042 Rennes, France

E-mail addresses

cvassall@laas.fr (C. Vassallo),

anne-helene.olivier@univ-rennes2.fr (A.H. Olivier),

soueres@laas.f r, (P.Souères),

armel.cretual@univ-rennes2.fr (A.Crétual),

ostasse@laas.fr (O. Stasse),

julien.pettre@inria.fr (J. Pettré)

Corresponding Author:

Anne-Hélène Olivier

E-mail: anne-helene.olivier@univ-rennes2.fr 
Tel. : +33299847138

Address: M2S lab, University Rennes 2 - ENS Rennes - UEB, Avenue Robert Schuman, Campus de Ker Lann, 35170 Bruz, France

Highlights

- A mobile robot was programmed to reproduce the interaction rules of human walkers

- We observed the behavior of human walkers crossing the way of this reactive robot

- Contrary to what occurs with a passive robot, the crossing order is preserved

- Humans behave with the reactive robot as when crossing another human walker

- Making robots move in a human-like way eases their interaction with human walkers

Wordcount is 2997 words.

\begin{abstract}
Previous studies showed the existence of implicit interaction rules shared by human walkers when crossing each other. Especially, each walker contributes to the collision avoidance task and the crossing order, as set at the beginning, is preserved along the interaction. This order determines the adaptation strategy: the first arrived increases his/her advance by slightly accelerating and changing his/her heading, whereas the second one slows down and moves in the opposite direction. In this study, we analyzed the behavior of human walkers crossing the trajectory of a mobile robot that was programmed to reproduce this human avoidance strategy. In contrast with a previous study, which showed that humans mostly prefer to give the way to a non-reactive robot, we observed similar behaviors between human-human avoidance and human-robot avoidance when the robot replicates the human interaction rules. We discuss this result in relation with the importance of controlling robots in a human-like way in order to ease their cohabitation with humans.
\end{abstract}

Keywords: Human-robot interaction; Locomotion; Collision avoidance; Gait adaptation; Mobile robot

\title{
Introduction
}

In everyday life, we walk by constantly adapting our motion to our environment. In past work, the relation between the walker and the environment was modeled as a coupled dynamical system. The trajectories result from a set of forces emitted by goals (attractors) and obstacles (repellers) [17]. Collision avoidance between pedestrians has also received a lot of attention either using front-on [3] or side-on approach trajectories $[7,8,11,12]$. Olivier et al. showed that walkers adapt their trajectory only if a future risk of collision exists [11]. This adaptation depends on the order of arrival of 
pedestrians that defines their order of passage. The first walker that arrives maintains or increases his/her advance by slightly accelerating and changing his/her direction to move away from the other participant. The second one slows down and moves in the opposite direction to reduce the risks of a collision. Huber et al. focused on how trajectories are adapted using speed and heading modifications depending on the crossing angle [7]. Future crossing order (who is about to give way or pass first) is quickly and accurately perceived and preserved until the end of the interaction $[8,12]$. This shows that walkers take efficiency into account since an inversion of the crossing order would result in suboptimal adaptations of higher amplitude. In addition, it was shown that the participant giving way contributes more to solving the collision avoidance [12]. Finally, behavior is influenced by the number of pedestrians to interact with and the potential to have social interactions with them [3].

Because humans and robots will have to share the same environment in the near future [5,9], recent studies focused on tasks involving walkers and a moving robot. Vassallo et al. [16] performed an experiment in which participants had to avoid collision with a passive wheeled robot (moving straight at constant speed), crossing perpendicularly their direction. In contrast to a human-human interaction, several inversions of the crossing order were observed, even though this behavior was not optimal. Such a behavior was observed when the walker arrived ahead of the robot with a predictable future crossing distance between 0 and $0.6 \mathrm{~m}$ but, despite this advance, finally gave way. This result was linked to the notion of perceived danger and safety, and to the lack of experience of interacting with such a robot.

Because of its design, the main limitation of Vassallo et al. study [16] was its inability to conclude whether the modification of the walker behavior was due to the lack of adaptability of the moving obstacle or solely to its artificial nature. Nonetheless, it was shown in [15] that the robot trajectory can be read and understood by humans in a task where a robot moves towards a human to initiate a conversation based on an approach linked to public and social distances. Furthermore, in a face-toface task with a moving robot, humans behave similarly whether they are told or not what the robot trajectory will be [1], showing their ability to actually read the robot motion.

Given these results, the question addressed in this paper is: "How would humans behave if they have to cross the trajectory of a robot programmed to replicate the observed human-human avoidance strategy?" Would humans understand that the robot adapts its trajectory and then adapt their own strategy accordingly, or would they give way to the robot as observed in [16]?

\section{Materials and methods}

\section{Participants}

Ten volunteers participated in the experiment ( 2 women and 8 men). They were $28.8( \pm 9.5)$ years old and $1.77 \mathrm{~m}$ tall $( \pm 0.12)$. They had no known pathology that could affect their locomotion. All of them had normal or corrected sight and hearing. All participants were naïve to the studied situation. Participants gave written and informed consent before their inclusion in the study. The experiment conformed to the Declaration of Helsinki, with formal approval of the ethics evaluation committee of INSERM (IRB00003888, Opinion number 13-124), Paris, France (IORG0003254, FWA00005831).

\section{Apparatus}


The experiment took place in a $40 \mathrm{~m} \times 25 \mathrm{~m}$ gymnasium. The room was separated into two areas by $2 \mathrm{~m}$ high occluding walls forming a gate in the middle (Figure1). Four specific positions were defined: the participant starting position PSP, the participant target PT, and two robot starting positions RSP1 and RSP2, to generate situations where the robot approached from the right or from the left of the participants. Two virtual guidelines $r_{a}$ and $r_{b}$, parallel to the line (RSP1, RSP2) and respectively located at a distance of $0.5 \mathrm{~m}$ and $1.0 \mathrm{~m}$ from the gate, were used as reference for guiding the robot to pass behind or ahead the participant during the avoidance phase. A specific zone between PSP and the gate was named Motion Estimation Zone (MEZ), far enough from PSP to let the participants reach their comfort velocity before they entered the MEZ. The intersection point between the robot and the initial path of the participant was named Hypothetical Crossing Point (HCP) as this is the point where the participant and robot would cross if they do not modify their trajectory.

Insert figure 1 here

\section{Task}

Participants were asked to walk at their preferred speed from PSP to PT passing through the gate. They were told that a robot could be moving beyond the gate and could obstruct them, meaning that the robot could adapt its trajectory according to the participants' one. One experimental trial corresponded to one travel from PSP to PT. We defined $t_{\text {see, }}$, the time at which the participant passed through the gate and saw the robot moving, and $t_{\text {cross, }}$ the time of closest approach, when the human-robot distance was minimal (i.e., the "distance of closest approach"). The crossing configuration and the risk of future collision were estimated using the Signed Minimal Predicted Distance, noted smpd, which gives, at each time step, the future distance of closest approach if both the robot and the participant keep a constant speed and direction [16]. A variation of smpd means that the participant or/and the robot are performing adaptation. The sign of this function depends on who, between the participant and the robot, is going to pass first: positive if it is the participant and negative otherwise. A change of smpd sign means a switch of the future crossing order.

\section{Recorded data}

3D kinematic data was recorded using a 16 infrared cameras motion capture Vicon-MX system $(120 \mathrm{~Hz})$. Reconstruction was performed with Vicon-Blade and computations with Matlab (Mathworks ${ }^{\circledR}$ ). The global position of participants was estimated as the centroid of the reflective markers set on a helmet they were wearing. The stepping oscillations were filtered out by applying a Butterworth low-pass filter (2nd order, dual pass, $0.5 \mathrm{~Hz}$ cut-off frequency).

\section{Robot Behavior}

We used a RobuLAB10 robot from Robosoft (dimension: $0.45 \times 0.40 \times 1.42 \mathrm{~m}$, weight $25 \mathrm{Kg}$, maximum speed $\sim 3 \mathrm{~m} . \mathrm{s}^{-1}$ ). The robot reference point was the center of its base. The robot control sequence was the following (cf. Figure1): 1) The robot was at rest at RSP1 or RSP2. 2) The participant crossed MEZ, its arrival time at HCP was estimated. 3) The theoretical speed at which the robot should move to reach HCP at the same time as the participant was estimated. This speed was then further increased (resp. decreased) for the robot to arrive in advance (respectively lately) at $\mathrm{HCP}$, in order to match the expected smpd. This choice was done such that smpd values at $t_{\text {see }}$ were randomly distributed in $[-0.9 m ; 0.9 m] 4$ ) When the robot had to avoid the human, $2 \mathrm{~m}$ before reaching HCP, the robot adapted its trajectory by inserting a new way-point on its trajectory, in order to pass behind the walker along the line $r_{-} a$ or ahead the walker by moving along the line $r_{-} b$, depending on the 
sign of smpd at $t_{\text {see. }}$.5) When the avoidance phase was over, the robot was controlled to reach its final position.

\section{Experimental plan}

Each participant performed 30 trials. The robot starting position ( $50 \%$ from RSP1, $50 \%$ from RSP2) was randomized among the trials. To introduce variability, in 2 trials the robot did not move. The participants were not informed about the initial position of the robot nor about the possibility that the robot would not move on every trial. Only the 28 trials with potential adaptations were analyzed.

\section{Analysis}

The analysis focused on the time interval during which adaptation was performed. To this end, smpd was normalized in time by resampling the function at 100 intervals between $t_{\text {see }}$ (time $0 \%$ ) and $t_{\text {cross }}$ (time 100\%). The quantity of adaptation was defined as the absolute value of the difference between $\operatorname{smpd}\left(t_{\text {see }}\right)$ (i.e., the initial conditions of the interaction) and $\operatorname{smpd}\left(t_{\text {cross }}\right)$ (i.e., the actual signed minimum distance between the participant and the robot).

Statistics were performed using Statistica $\left(\right.$ Statsoft $\left.^{\circledR}\right)$. All effects were reported at $p<0.05$. Normality was assessed using a Kolmogorov-Smirnov test. Depending on the normality, values are expressed as median $(M)$ or mean $\pm S D$. Wilcoxon signed-rank tests were used to determine differences between values of smpd at $t_{\text {see }}$ and $t_{\text {cross. }}$. The influence of the crossing order evolution on the smpd values was assessed by using a Kruskal-Wallis test with post hoc Mann-Whitney tests for which a Bonferroni correction was applied: all effects are reported at a 0.016 level of significance (0.05/3). Finally, we used a Mann-Whitney test to compare the crossing distance depending on the final crossing order.

\section{Results}

We considered 279 trials (one has been removed because the robot failed to start). Figure 2 depicts the evolution of smpd for all trials.

Insert figure 2 here

The sign of smpd at $t_{\text {cross }}$ showed that participants passed first in $53 \%$ of cases, and gave way in the other $47 \%$. Combining this information with the data at $t_{\text {see, }}$ we could evaluate if an inversion of crossing order occurred. The trials have been divided into 4 categories, depending on the relative signs of smpd at $t_{\text {see }}$ and $t_{\text {cross }}$ (Pos for positive and Neg for negative): PosPos, PosNeg, NegPos, NegNeg. For example, the PosNeg category contained the trials for which $\operatorname{smpd}\left(t_{s e e}\right)>0$ and $\operatorname{smpd}\left(t_{\text {cross }}\right)<0$.

smpd categories were distributed among the trials in the following way: PosPos=144 trials (52\%), NegNeg=110 trials (39\%), PosNeg=22 trials (8\%), NegPos=3 trials (1\%). All participants had both PosPos and PosPos trials, and 9 out of 10 participants had at least one PosNeg trial. In the remainder of the paper, the NegPos category will not be further considered as it contained only three trials defined as outliers. Examples of corresponding trajectories for each of the 3 remaining categories are depicted in Figure3. Note that in $91 \%$ of cases the crossing order was preserved. We only observed 
$9 \%$ of trials where participants were likely to pass first but adapted their trajectory to finally give way to the robot.

Insert figure 3 here

Figure $4 a$ and $4 \mathrm{~b}$ show respectively the average evolution of smpd and its time derivative for each category. Based on the sign of the smpd time derivative, we can separate the reaction period during which participants perform adaptations (smpd varies) from the regulation period that follows the collision avoidance (the derivative vanishes, and its sign may even change) as defined in [8] and [16]. The relative duration of the reaction phase for PosPos (55\%) and NegNeg (57\%) trials was almost the same, while participants were longer to adapt when they decided to give way to the robot in PosNeg (69\%) trials.

Insert figure 4 here

Figure 5 shows comparison between $\operatorname{smpd}\left(t_{\text {see }}\right)$ and $\operatorname{smpd}\left(t_{\text {cross }}\right)$ for the 3 categories. For each category, the human-robot distance increased from $t_{\text {see }}$ to $t_{\text {cross }}$ so that the risk of collision was reduced. Statistical analysis showed a significant difference of smpd between $t_{\text {see }}$ and $t_{\text {cross }}$ for PosPos trials (Msmpdtsee $=0.71 \mathrm{~m}$, Msmpdtcross=1,08m, $Z=9.17, p<0.0001, r=0.76$ ), for NegNeg trials (Msmpdtsee $=-0.46 \mathrm{~m}$, Msmpdtcross $=-1.14 \mathrm{~m}, \mathrm{Z}=8.98, \mathrm{p}<0.0001, \mathrm{r}=0.85$ ) and for PosNeg trials (Msmpdtsee $=0.29 \mathrm{~m}$, Msmpdtcross $=-0.71 \mathrm{~m}, \mathrm{Z}=4.11, \mathrm{p}<0.0001, \mathrm{r}=0.88$ ).

Insert figure 5 here

Finally, the distance of closest approach was influenced by the category $(H(2,276)=29.3, p<0.0005)$. Post-hoc tests showed that the median distance between the robot and participants did not significantly differ between PosPos $(M=1.08 \mathrm{~m})$ and NegNeg $(M=1.14 \mathrm{~m})$ trials. However, when an inversion of the crossing order in PosNeg trials occurred, this distance was smallest $(M=0.71 \mathrm{~m})$.

\section{Discussion}

In the current study, results indicated that when a human is crossing the trajectory of a mobile robot which is programmed to replicate the observed human avoidance strategy, strong characteristics of collision avoidance are comparable with the ones of human-human interactions. First, the crossing order is preserved from $t_{\text {see }}$ to $t_{\text {cross }}$ in a majority of trials, as observed in human-human interactions $[8,11]$. However, in $8 \%$ of trials, the participants gave way to the robot while they were in position to pass first. Such a behavior was observed when $\operatorname{smpd}\left(t_{\text {see }}\right)$ was around $0.39 \mathrm{~m}$. Above this threshold, participants preferred to preserve their role rather than giving way to the robot. This result is confirmed by the repartition into PosPos and PosNeg categories of trajectories starting from the smpd interval $[0.39 \mathrm{~m}, 0.74 \mathrm{~m}]$, where $94 \%$ of trials belong to the PosPos group. This result is in contrast with the one previously observed with a passive robot [16], where participants consistently preferred to give way to the robot when the risk of collision was below $0.81 \mathrm{~m}$, even though this choice was not optimal. Note that, whether or not an inversion of the crossing order occurred, the trajectories were adapted in order to increase the crossing distance between the human and the robot to reduce the risk of collision. Results show that humans solve the collision avoidance with anticipation, as previously demonstrated during human-human interaction [11]. Indeed, Figure 4 
shows a plateau in smpd values before $t_{\text {cross }}$ meaning that the avoidance maneuvers are over before the end of the task. As discussed in the review of Higuchi [6], the anticipatory nature of adaptive locomotor strategies ensures safety navigation during the task. When the participant decides to preserve the crossing order, the task is solved earlier than when a switch of roles occurs, that requires more motion adaptation.

The human-human avoidance strategy takes advantage from the configurations of both agents to limit their adaptations $[11,12]$. Assuming that both participants have similar locomotion capabilities, a role is assigned to each of them depending on their order of passage, as recalled in the introduction. This high-level strategy is not related to the anthropomorphic walking, it is simply expressed in terms of the trajectory of a representative point (e.g. the waist position and heading) in the horizontal plane of motion. As such, the method can be easily transferred to a wheeled robot. The fact that the robot automatically initiates its avoidance motion by replicating the human strategy allows the human to easily go back to the process usually applied. In this way, the human easily understands the role he/she should play and no conflicting situation occurred in any trials. For this reason, our overall results are comparable to previous findings, which were reported in the case of a human-human interaction.

The control of our robot follows a model of shared-avoidance strategy based on the human behavior [16]. One conflicting situation might theoretically occur when both agents arrive with a zero smpd (i.e. exactly at the same time) and take the same role. Such a conflicting situation between human walkers was not reported in [16] and never occurred in our human-robot experiment. When the human and the robot were approaching the crossing point quite simultaneously, the smpd was checked twice: once at the beginning, based on the measure of the human velocity in the MEZ, and once at $t_{\text {see. }}$. Based on this accurate measurement of the smpd, which is never exactly equal to zero, the robot adopts a role that helps the walker adapt his behavior. For this reason, we never observed any conflicting situation in which the walker would have tried to force the way (NegPos) after the robot had initiated the avoidance. However, the opposite situation (PosNeg), in which the human prefers to give way to the robot though he arrived ahead, was sometimes observed. This cautious behavior does not constitute a conflicting situation that could block both agents.

The behavioral similarities observed between human-human and human-robot is in accordance with the study of Carton et al. [1], in which a walker avoids a robot that reproduces an average human trajectory to avoid a face-to-face collision. They showed that giving a human-like behavior to a moving robot gives rise to readable motions that convey intentions. This readability allows humans to minimize their planning effort and avoid the collision earlier and smoothly. In accordance with previous studies $[1,2,10]$, our result shows that controlling robots in order to make them behave in a human-like way is a key point to ease human-robot cohabitation.

\section{Conclusion}

Our study suggests that when human walkers cross the trajectory of a mobile robot that obeys the observed human-human avoidance rules, they behave closely as when they cross the trajectory of another human walker. This result shows that, for the ease of human-robot collaboration, machines should move by respecting human interaction rules.

In future works, as previously investigated in human-human interactions $[3,4,6,13]$, it would be interesting to better understand the visual anticipation processes as well as the nature of the visual information underlying such a collaboration. This can be done by using an eye-tracking system to 
couple the adaptations made by the human walkers and the gaze-activity. Also, it would be interesting to evaluate whether the use of a humanoid robot, whose morphology is closer to the one of a human than a wheeled robot, modifies the human behavior. Another direction of research would be to extend this work to the case of multiple walkers interacting with each other at the same time. Would it be possible, if some participants are replaced by robots that behave like humans, to observe the same human adaptation? Finally, the nature of human expectations and presuppositions, that can be linked to the notion of socially-aware navigation (see [14] for a review), should have strong influence on the walker behavior. Indeed, in a less controlled context, participants would certainly behave differently than in the framework of a scientific experiment, where the robot is expected to behave safely. An interesting complement of study would then be to lead similar experiment in real-life environment to evaluate the impact of the context to the walker behavior.

Conflict of interest

In this work, there are no conflict of interest.

\section{Acknowledgements}

The research leading to these results has received funding from the European Union Seventh Framework Programme (FP7/2007 - 2013) under grant agreement n. 611909 (KoroiBot) and from the French National Research Agency, projects Entracte (\#ANR-13-CORD-0002) and Percolation (\#ANR13-JS02-0008). 


\section{BIBLIOGRAPHY}

[1] D. Carton, W. Olszowy, D. Wollherr, Measuring the effectiveness of readability for mobile robot locomotion, Int. J. Soc. Robot. 8 (2016) 721-741.

[2] A. Dragan, S. Srinivasa, Generating legible motion, In: Robotics: Science and Systems (2013).

[3] Dicks, M., Clashing, C., O'Reilly, L., \& Mills, C. Perceptual-motor behaviour during a simulated pedestrian crossing. Gait \& Posture 49 (2016), 241-245.

[4] Gallup, A. C., Hale, J. J., Sumpter, D. J., Garnier, S., Kacelnik, A., Krebs, J. R., \& Couzin, I. D. Visual attention and the acquisition of information in human crowds. Proceedings of the National Academy of Sciences, 109 (2012) 7245-7250.

[5] M.A. Goodrich, A.C. Schultz, Human-robot interaction: a survey, Found. Trends Hum. Comput. Interact. 1 (2007) 203-275.

[6] Higuchi, T. Visuomotor Control of Human Adaptive Locomotion: Understanding the Anticipatory Nature. Frontiers in Psychology, 4, (2013) 277, 1-9.

[7] M. Huber, Y.-H. Su, M. Krüger, K. Faschian, S. Glasauer, J. Hermsdörfer, Adjustments of speed and path when avoiding collisions with another pedestrian, PLoS One 9 (2014).

[8] A.G. Knorr, L. Willacker, J. Hermsdörfer, S. Glasauer, M. Krüger, Influence of Person-and SituationSpecific Characteristics on Collision Avoidance Behavior in Human Locomotion. J. Exp Psychol.: Hum. Percept. Perform. 42 (2016) 1332-1343.

[9] T. Kruse, A.K. Pandey, R. Alami, A. Kirsch, Human-aware robot navigation: a survey, Rob. Auton. Syst. 61 (2013) 1726-1743.

[10] C. Lichtenthäler, A. Kirsch, Towards legible robot navigation-how to increase the intend expressiveness of robot navigation behavior, in: Int. Conf. Soc. Robot. Embodied Commun. Goals Intentions, 2013.

[11] A.H. Olivier, A. Marin, A. Crétual, J. Pettré, Minimal predicted distance: A common metric for collision avoidance during pairwise interactions between walkers, Gait Posture 36 (2012) 399-404.

[12] A.-H. Olivier, A. Marin, A. Crétual, A. Berthoz, J. Pettré, Collision avoidance between two walkers: role-dependent strategies., Gait Posture 38 (2013) 751-6.

[13] Passos, P., Araújo, D., Davids, K., Gouveia, L., Milho, J., \& Serpa, S. Information-governing dynamics of attacker-defender interactions in youth rugby union. Journal of Sports Sciences 26 (2008), 1421-1429.

[14] Rios-Martinez, J., Spalanzani, A., \& Laugier, C., From proxemics theory to socially-aware navigation: A survey. International Journal of Social Robotics, 7 (2015), 137-153.

[15] S. Satake, T. Kanda, D.F. Glas, M. Imai, H. Ishiguro, N. Hagita, How to Approach Humans? Strategies for Social Robots to Initiate Interaction, in: Proc. 4th ACM/IEEE Int. Conf. Hum. Robot Interact., ACM, New York, NY, USA, (2009) 109-116.

[16] C. Vassallo, A.-H. Olivier, P. Souères, A. Crétual, O. Stasse, J. Pettré, How do walkers avoid a mobile robot crossing their way? Gait Posture 51 (2017) 97-103.

[17] W.H. Warren, B.R. Fajen, Behavioral dynamics of visually guided locomotion, Coordination: neural, behavioral and social dynamics (2008) 45-75. 
Figure 1: Experimental apparatus and task. The robot moves from RSP1 to RSP2 (or vice versa), following the lateral path $r \_b$ or $r_{-} a$ to pass respectively behind or ahead the participant.

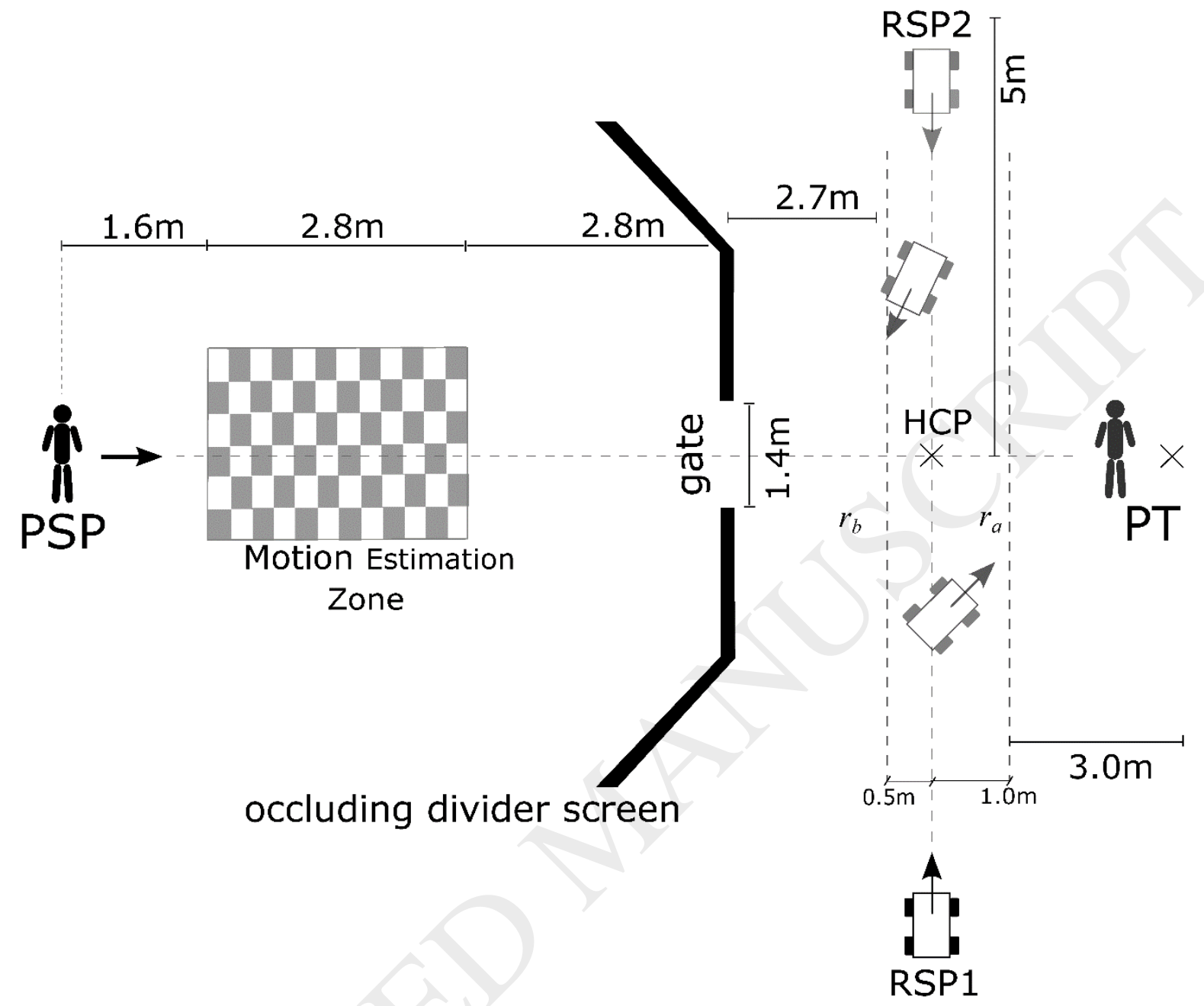

Figure 2: Evolution of smpd normalized in time during the interaction [ $\left.t_{\text {see }}, t_{\text {coss }}\right]$ for all the 279 trials. Gray curves represent trials where the initial crossing order was preserved while black curves represent trials where the initial crossing order was changed. 


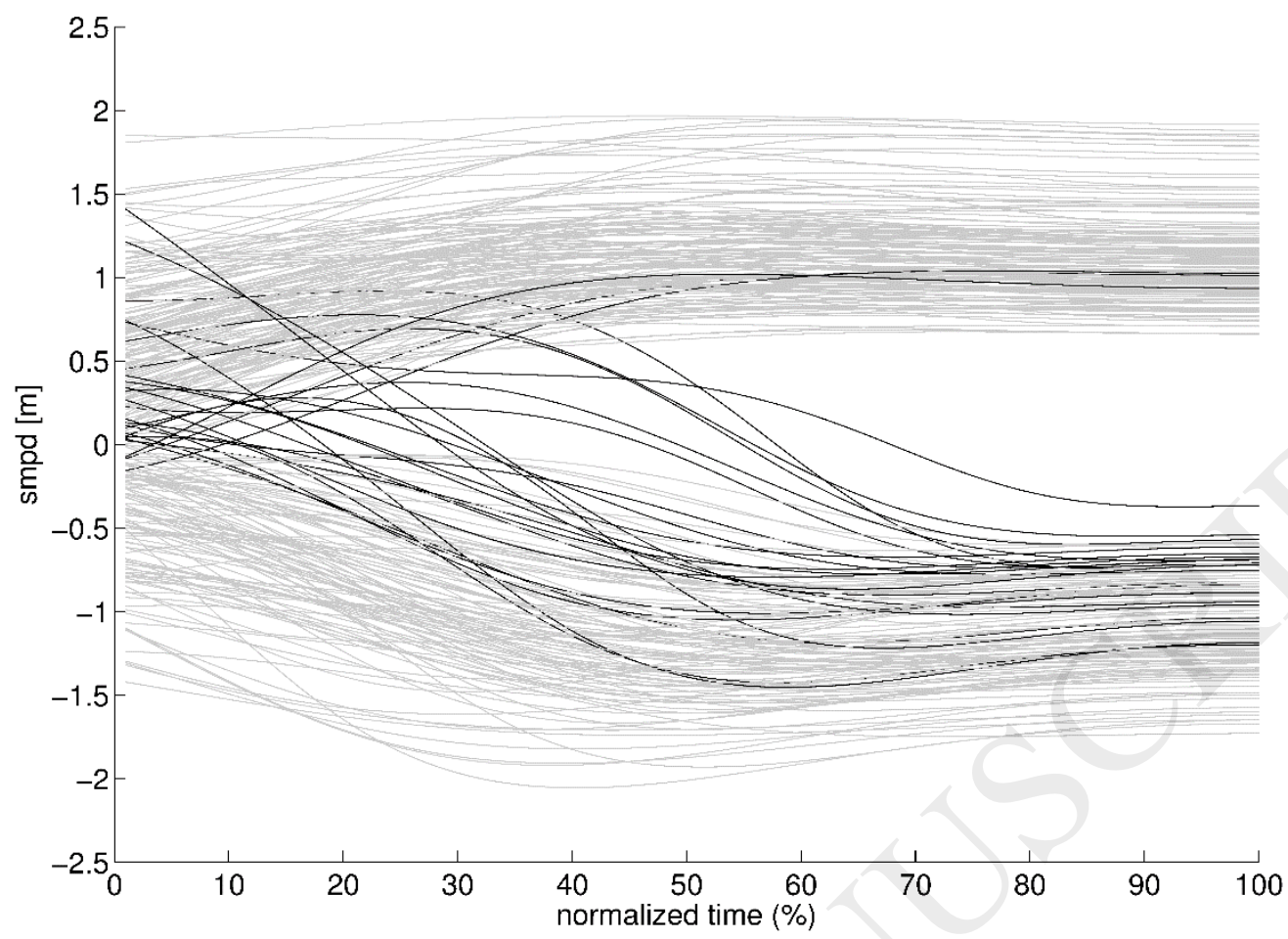

Figure 3: Three examples of participant's $(P)$ and robot $(R)$ trajectories during the interaction phase, for PosPos (top), PosNeg (middle) and NegNeg (bottom) categories. The part of the trajectory between $t_{\text {see }}$ (circle mark) and $t_{\text {cosss }}$ (square mark) is represented in bold line. Corresponding positions along time are linked by dotted lines. 


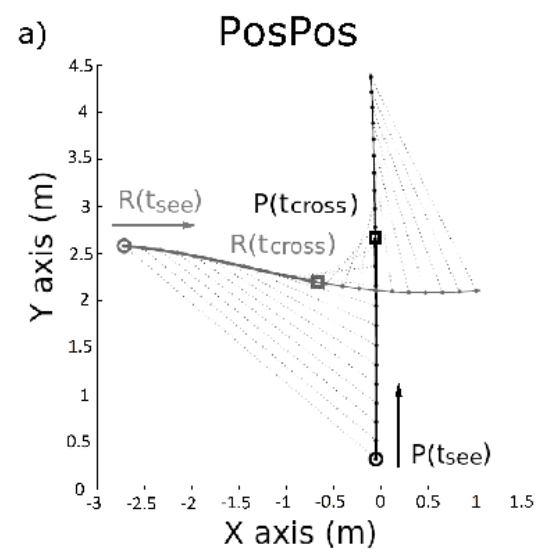

b) 5 . PosNeg
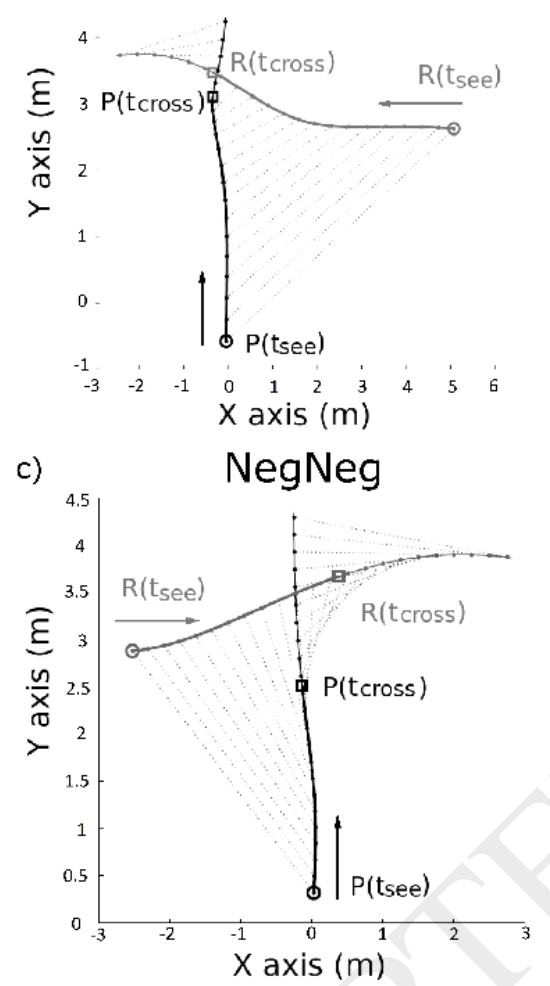

Figure 4: (a) Mean evolution ( $\pm 1 \mathrm{SD}$ ) of smpd for each category of trial. (b) Time derivative of the mean smpd. The three vertical segments correspond, for each curve (PosPos, PosNeg or NegNeg), to the time at which the time derivative of the smpd vanishes, i.e., separate the reaction phase (on the left) from the regulation phase (on the right). 


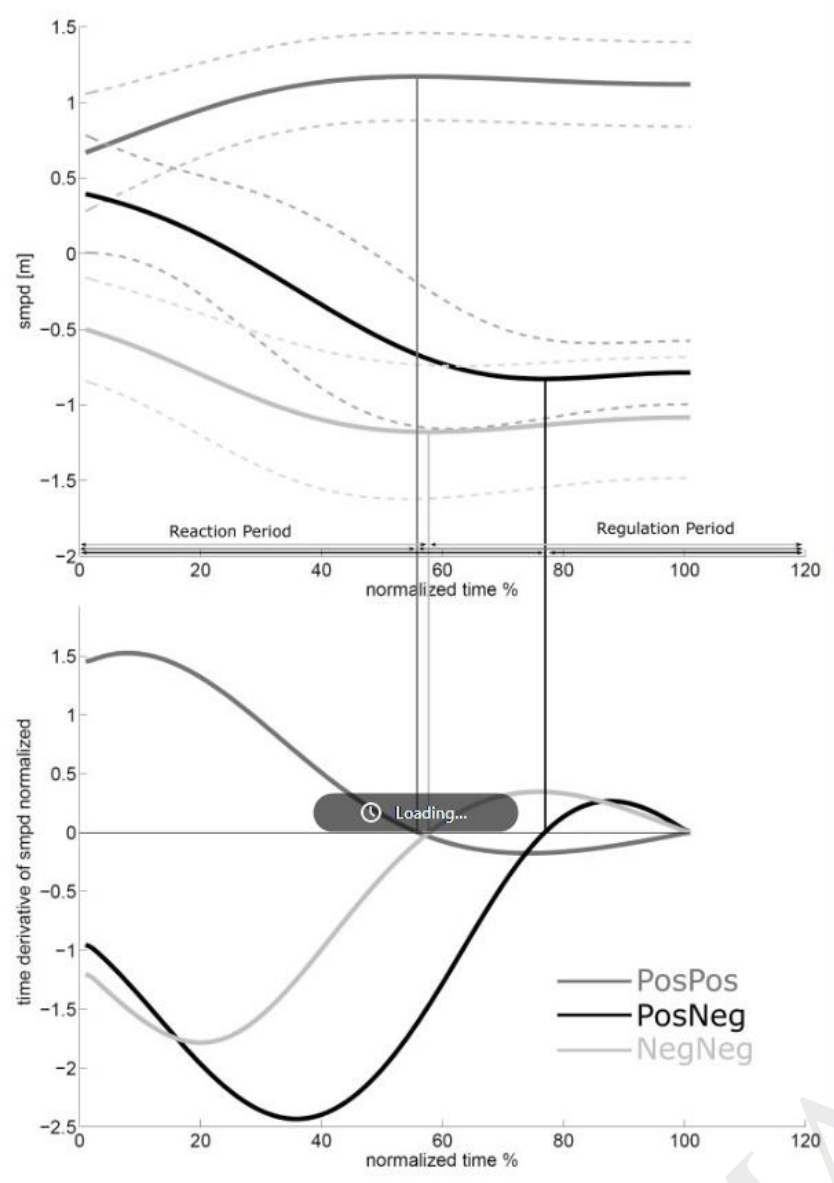

Figure 5: smpd values for PosPos, PosNeg and NegNeg categories at $t_{\text {see }}$ and $t_{\text {cosss. }}$ A significant difference in values means that adaptations were made to the trajectory by the participant $\left({ }^{* * *} p<0.001\right)$.

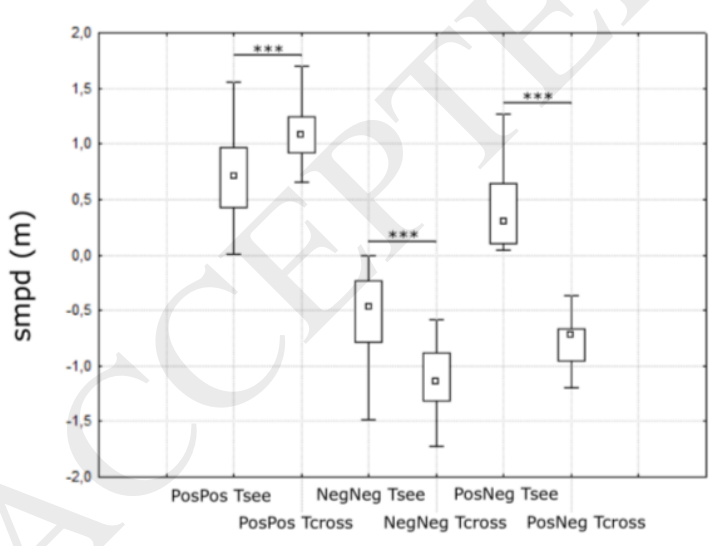

